\title{
Mechanistic target of rapamycin complex 1 signaling regulates cell proliferation, cell survival, and differentiation in regenerating zebrafish fins
}

\author{
Kentaro Hirose, Taishi Shiomi, Shunya Hozumi and Yutaka Kikuchi*
}

\begin{abstract}
Background: The mechanistic target of rapamycin complex1 (mTORC1) signaling pathway has been implicated in functions of multicellular processes, including cell growth and metabolism. Although recent reports showed that many signaling pathways, including Activin, Bmp, Fgf, sonic hedgehog, Insulin-like growth factor (IGF), Notch, retinoic acid, and Wnt, are implicated in non-mammalian vertebrate regeneration, also known as epimorphic regeneration, mTORC1 function remains unknown.
\end{abstract}

Results: To investigate the role of mTORC1 signaling pathway in zebrafish caudal fin, we examined the activation and function of mTORC1 signaling using an antibody against phosphorylated S6 kinase and a specific inhibitor, rapamycin. mTORC1 signaling is activated in proliferative cells of intra-ray and wound epidermal cells before blastema formation, as well as in proliferative blastema cells, wound epidermal cells, and osteoblasts during regenerative outgrowth. Before blastema formation, proliferation of intra-ray and wound epidermal cells is suppressed, but cell death is not affected by mTORC1 signaling inhibition with rapamycin. Moreover, rapamycin treatment inhibits blastema and wound epidermal cell proliferation and survival during blastema formation and regenerative outgrowth, as well as osteoblast proliferation and differentiation during regenerative outgrowth. We further determined that mTORC1 signaling is regulated through IGF-1 receptor/phosphatidylinositol-3 kinase and Wnt pathways during fin regeneration.

Conclusion: Taken together, our findings reveal that mTORC1 signaling regulates proliferation, survival, and differentiation of intra-ray cells, wound epidermis, blastema cells, and/or osteoblasts in various fin regeneration stages downstream of IGF and Wnt signaling pathways.

Keywords: Mechanistic target of rapamycin, Fin, Regeneration, Zebrafish, Osteoblast, Cell proliferation, Cell survival, Differentiation

\section{Background}

Mammalians present a limited ability for organ regeneration, whereas various non-mammalian vertebrates such as teleosts and urodele amphibians show outstanding regeneration ability. Among them, the zebrafish is a useful animal model, which has been used to study the regeneration of several organs or appendages [1,2]. The adult zebrafish caudal fin is composed of multiple cell types, including fibroblast-like mesenchymal cells, osteoblasts,

\footnotetext{
* Correspondence: yutaka@hiroshima-u.ac.jp

Department of Biological Science, Graduate School of Science, Hiroshima University, Kagamiyama 1-3-1, Higashi-Hiroshima, Hiroshima 739-8526, Japan
}

endothelial cells, neurons, and epidermal cells, and the fin regeneration process presents three stages: pre-blastema formation, blastema formation, and regenerative outgrowth [3-5]. Following fin amputation, epidermal cells migrate to cover the wound within 12 hours post amputation (hpa) [5]. The intra-ray mesenchymal cells and osteoblasts then migrate toward the amputation plane by 24 hpa (pre-blastema formation stage) [5]. From 18 to 24 hpa, these intra-ray mesenchymal cells and osteoblasts begin to proliferate [3] and, as a result, a population of these cells, named blastema, is formed underneath the wound epidermis by 48 hpa (blastema formation stage). 
After 48 hpa, regenerative outgrowth starts and the ray blastema mainly consists of three distinct domains: the distal blastema, proliferative zone, and differentiation zone (72 hpa) $[5,6]$. The distal blastema barely contains proliferative blastema cells, and the proliferative zone contains highly proliferative mesenchymal cells (the proximal medial blastema) and osteoblasts (72 hpa) $[3,5,6]$.

Since rapamycin presents various physiological functions such as antifungal, immunosuppressive, and antiproliferative properties, many researchers have focused on the identification of rapamycin intracellular targets [7]. Mechanistic target of rapamycin (mTOR), a serine/threonine kinase, has been shown to be a rapamycin target in yeast, and most eukaryotes have this protein $[7,8]$. The mTOR signaling pathway is mainly involved in cell growth and metabolism as two distinct complex types, mTOR complex 1 (mTORC1) and 2 (mTORC2) $[7,8]$. The mTORC1 signaling pathway is involved in multicellular processes, including protein synthesis, lipid synthesis, glycolysis, and autophagy, and is specifically inhibited by rapamycin $[7,8]$. mTORC1 signaling is known to regulate protein synthesis mainly through direct phosphorylation of S6 kinase (S6K) $[7,8]$.

Many signaling pathways, including Activin, Bmp, Fgf, sonic hedgehog, Insulin-like growth factor (IGF), Notch, retinoic acid, Wnt, and reactive oxygen species (ROS), are implicated in the regulation of cell proliferation and/or differentiation in non-mammalian vertebrate regeneration, also known as epimorphic regeneration [2,9-12]. However, the spatiotemporal activation and function of the mTORC1 signaling pathway during epimorphic regeneration remains unknown. In this study, we explored the activation and function of mTORC1 signaling during various stages of zebrafish caudal fin regeneration, and identified the upstream signaling pathway leading to mTORC1 signaling activation during caudal fin regeneration.

\section{Results \\ Spatiotemporal dynamism of mTORC1 signaling activation during fin regeneration}

To investigate the molecular mechanisms of regeneration, we analyzed the signaling pathways involved in zebrafish fin regeneration using various inhibitors and drugs. Our experiments indicated that rapamycin, a well-known inhibitor of mTORC1 signaling, presented a strong inhibitory effect on fin regeneration. To analyze the activation of mTORC1 signaling during fin regeneration, spatiotemporal distribution of phosphorylated S6 kinase (p-S6K), an activated form of S6K, was first examined by immunohistochemistry. Although no p-S6K-positive cells were found in intra-ray and epidermal cells immediately after fin amputation (0 hpa) (Figure 1A), p-S6K signals were detected as early as $6 \mathrm{hpa}$ (Figure 1B). The number of p-S6Kpositive cells in the intra-ray, epidermis, and blastema was markedly increased, and p-S6K-positive cell localization gradually changed with the progression of fin regeneration. These p-S6K-positive cells were widely distributed in the intra-ray and wound epidermis proximal to the amputation plane from 6 to $18 \mathrm{hpa}$ (arrowheads in Figure 1B-D). From $24 \mathrm{hpa}$, these cells started to accumulate underneath the wound epidermis, and p-S6K-positive blastema cells were evident at 36 hpa (arrowheads in Figure 1E,F). After $48 \mathrm{hpa}$, p-S6K-positive cells were mainly detected in the blastema and wound epidermis. At 72 and 120 hpa, p-S6K signals were restricted to three distinct domains in the blastema, the putative proximal medial blastema domain (arrowheads in Figure 1H,I') [3], the bilateral stripped-domains (arrows in Figure 1H,I'), where differentiating osteoblasts and their progenitor cells are localized $[11,13]$, and the wound epidermis. On the other hand, it is interesting to note that p-S6K signals were absent in the tip of the putative proximal medial blastema domain and putative distal blastema (brackets in Figure 1G, $\left.\mathrm{H}, \mathrm{I}^{\prime}\right)$. These results suggest that, although the mTORC1 signaling pathway is widely activated in the intra-ray, wound epidermal cells, and blastema cells until $48 \mathrm{hpa}$, mTORC1 signaling is gradually restricted to the putative proliferative blastema cells and osteoblasts after $72 \mathrm{hpa}$.

To characterize $\mathrm{p}$-S6K-positive cells during the preblastema formation ( $24 \mathrm{hpa}$ ) and regenerative outgrowth stages (72 hpa), the fin regenerates were co-immunostained with proliferating cell nuclear antigen (PCNA), a marker for proliferative cells [14]; Zns-5, a marker for all osteoblasts independent of differentiation stages [15]; or Runx2, an osteoblast progenitor marker [16]. At $24 \mathrm{hpa}$, almost all p-S6K-positive intra-ray and blastema cells were PCNA-positive (Figure 2A-C'), and all Runx2-positive osteoblast progenitors were p-S6K-positive (Figure 2D-F'), suggesting that mTORC1 signaling is active in proliferative cells and osteoblast progenitors during the preblastema formation stage. At 72 hpa, PCNA-positive cells in the putative proximal medial blastema domain were p-S6K-positive (arrowheads in Figure 2I'), except that the tip of the putative proximal medial blastema domain (a bracket in Figure 2I') was p-S6K-negative. In the bilateral-stripped domains, Zns-5- or Runx2-positive cells were p-S6K-positive (arrowheads in Figure 2L',O'), except in the most distal regions of these domains (brackets in Figure 2L',O'). A recent report showed that Runx2-positive self-renewing preosteoblasts are localized in the most distal region and that Runx2/Sp7 (Osterix) double-positive cells are differentiating osteoblasts [11]. Our results suggest that mTORC1 signaling is active in proliferative blastema cells and differentiating osteoblasts during the regenerative outgrowth stage. These spatiotemporal mTORC1 activation patterns prompted us to further analyze the function of mTORC1 signaling in the preblastema formation, blastema formation, and regenerative outgrowth stages. 


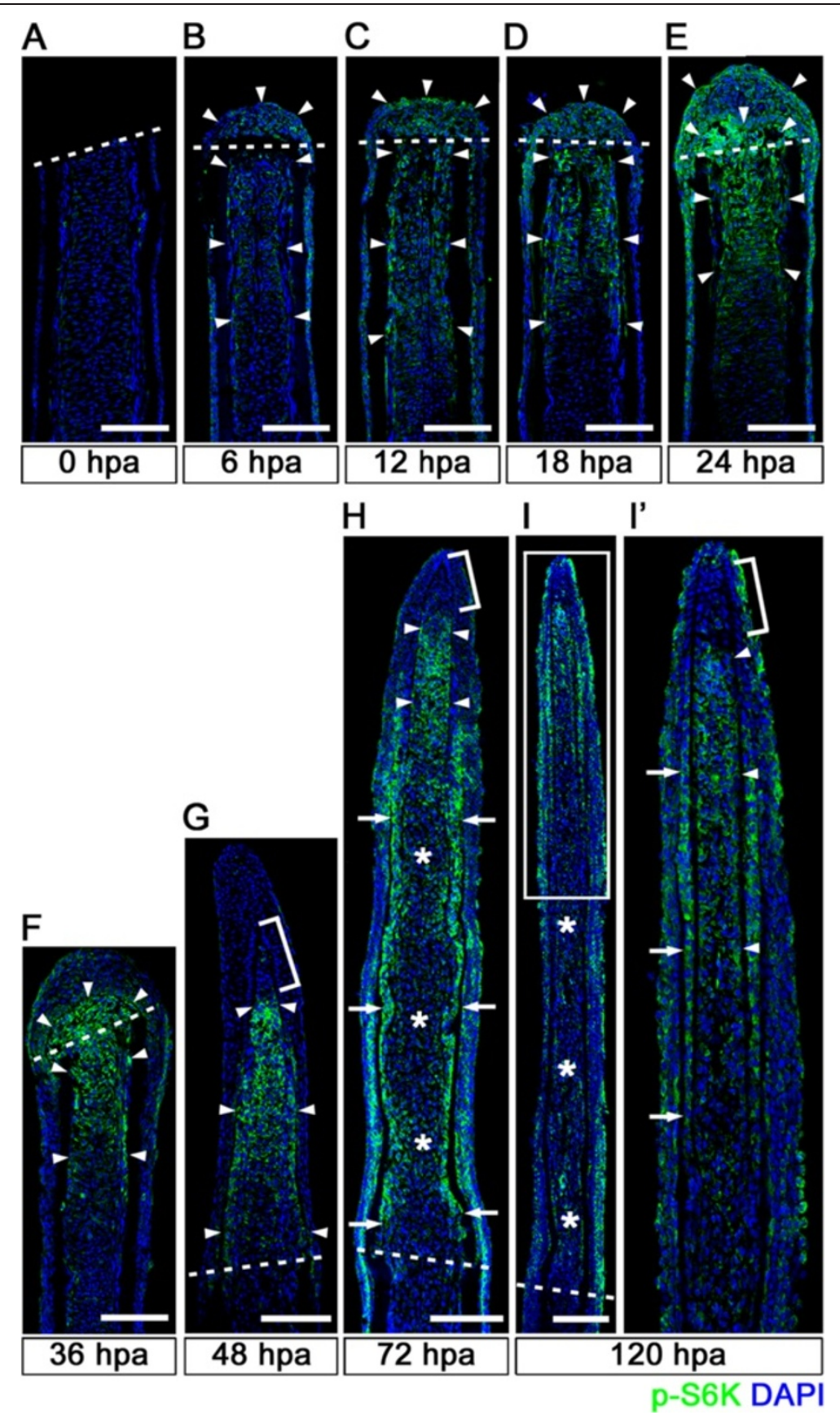

Figure 1 Spatiotemporal activation of S6K during zebrafish fin regeneration. (A-I') Longitudinal sections of wild-type fin regenerates that were immunohistochemically stained with an antibody against p-S6K (green) at 0 (A), 6 (B), 12 (C), 18 (D), 24 (E), 36 (F), 48 (G), 72 (H), and 120 (I,I') hpa (0 hpa, $\mathrm{n}=3 ; 6$ hpa, $\mathrm{n}=4 ; 12 \mathrm{hpa}, \mathrm{n}=3 ; 18 \mathrm{hpa}, \mathrm{n}=4 ; 24 \mathrm{hpa}, \mathrm{n}=5 ; 36 \mathrm{hpa}, \mathrm{n}=4 ; 48 \mathrm{hpa}, \mathrm{n}=3 ; 72 \mathrm{hpa}, \mathrm{n}=4 ; 120 \mathrm{hpa}, \mathrm{n}=3$ ). The boxed area in I is enlarged in I'. DAPI fluorescent signal (blue) indicates the presence of nuclei. Dashed white lines indicate the amputation plane. The p-S6K fluorescent signals were barely detectable in the amputated fin at $0 \mathrm{hpa}$ (A). At $6 \mathrm{hpa}$, p-S6K-positive cells were found in both intra-ray and epidermal cells (arrowheads in B), and the number of p-S6k-positive cells increased by 24 hpa (arrowheads in C, D, and E). Although p-S6Kpositive cells were found in both the blastema and intra-ray region adjacent to and proximal to the amputation plane at $36 \mathrm{hpa}$ (arrowheads in F), p-S6K-positive cells were mainly detected in the blastema at 48 hpa (arrowheads in G). At 72 and 120 hpa, p-S6K-positive cells were observed in the bilateral strip regions (arrows in $\mathbf{H}$ and $\mathbf{I}^{\prime}$ ), in the putative proximal medial blastema (arrowheads in $\mathbf{H}$ and $\mathbf{I}^{\prime}$ ), and in the wound epidermis, but not in the putative differentiated blastema cells (asterisks in $\mathbf{H}$ and $\mathbf{I}$ ). Brackets indicate the p-S6K-negative cells in the tip of the putative proximal medial blastema domain and putative distal blastema $\left(\mathbf{G}, \mathbf{H}, \mathbf{I}^{\prime}\right)$. It should be noted that both p-S6K and DAPI fluorescent signals did not overlap, as p-S6K and genomic DNA (DAPI specifically stains double-strand DNA) are localized in the cytosol and nucleus, respectively. Scale bars: $100 \mu \mathrm{m}$. 


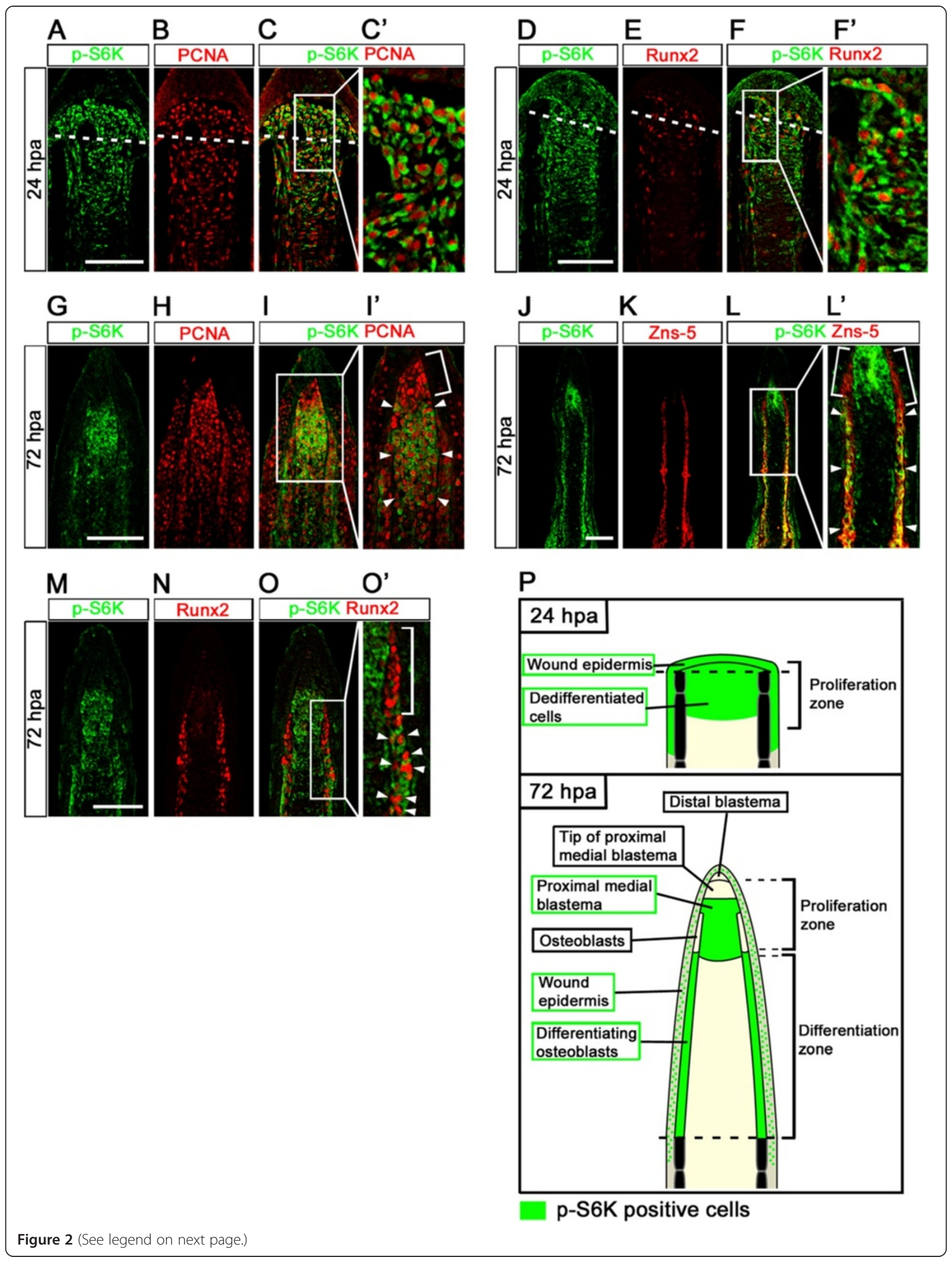


(See figure on previous page.)

Figure 2 Distributions of S6K-positive cells and proliferative cells at $\mathbf{2 4}$ and $\mathbf{7 2} \mathrm{hpa}$. (A-F') Longitudinal sections of wild-type fin regenerates that were co-immunohistochemically stained with antibodies against p-S6K (green) and PCNA (red) $\left(\mathbf{A}-\mathbf{C}^{\prime}, n=3\right)$ or p-S6K (green) and Runx2 (red) $\left(\mathbf{D}-\mathbf{F}^{\prime}, \mathbf{n}=3\right)$ at 24 hpa. p-S6K positive cells were PCNA- or Runx2-positive at $24 \mathrm{hpa}\left(\mathbf{C}^{\prime}, \mathbf{F}^{\prime}\right)$. The boxed areas in $\mathbf{C}$ and $\mathbf{F}$ are enlarged in $\mathbf{C}^{\prime}$ and $\mathbf{F}^{\prime}$, respectively. Dashed white lines indicate the amputation planes. (G-O') Longitudinal sections of wild-type fin regenerates that were co-

immunostained with antibodies against p-S6K (green) and PCNA (red) (G-I', n=5), p-S6K (green) and Zns-5 (red) $\left(\mathbf{J}-\mathbf{L}^{\prime}, \mathrm{n}=3\right.$ ), or p-S6K (green) and Runx2 (red) $\left(\mathbf{M}-\mathbf{O}^{\prime}, n=4\right)$ at $72 \mathrm{hpa}$. PCNA-positive cells were p-S6K-positive in the putative proximal medial blastema domain (arrowheads in $\mathbf{I}^{\prime}$ ), but not in the tip of the putative proximal medial blastema domain (a bracket in $\mathbf{I}^{\prime}$ ). Zns-5- or Runx2-positive cells in the proximal lateral blastema were also p-S6K-positive (arrowheads in $\mathbf{L}^{\prime}$ and $\mathbf{O}^{\prime}$ ), but cells in the distal region of the lateral blastema were not (brackets in $\mathbf{L}^{\prime}$ and $\mathbf{O}^{\prime}$ ). It should be noted that both p-S6K and PCNA or Runx2 fluorescent signals did not overlap, because p-S6K and PCNA or Runx2 are localized in the cytosol and nucleus, respectively. Scale bars: $100 \mu \mathrm{m}$. (P) Cartoon summarizing the anatomical structures of fin regenerates in longitudinal cross-sections and localization of p-S6K-positive cells in the fin regenerates at 24 and $72 \mathrm{hpa}$. Dashed lines indicate the amputation planes.

mTORC1 signaling is required for cell proliferation, but not for cell survival during the pre-blastema formation stage To examine the function of mTORC1 signaling during fin regeneration, adult zebrafish were treated with a specific mTORC1 inhibitor, rapamycin, $12 \mathrm{~h}$ before amputation $(-12 \mathrm{~h})$ to $72 \mathrm{hpa}$ (Figure 3A). Rapamycin significantly inhibited fin regeneration compared to DMSO (Figure 3B, C). In addition to rapamycin, we examined the two different pharmacological inhibitors, Torin1 [17] and AZD8055 [18], in mTOR signaling inhibition, and found that fin regeneration was also significantly inhibited by both Torin1 and AZD8055 treatment (Additional file 1: Figure S1). mTORC1 signaling inhibition, by rapamycin, Torin1, and AZD8055 was confirmed by the loss of p-S6K signal at 72 hpa (Additional file 2: Figure S2). The p-S6K signals were markedly reduced by $3 \mathrm{~h}$ treatment with these inhibitors (rapamycin, Torin1, and AZD8055) and were nearly diminished by $6 \mathrm{~h}$ treatment (Additional file 3: Figure S3), suggesting the specificity of $\mathrm{p}-\mathrm{S} 6 \mathrm{~K}$ as readout of mTORC1 signaling. Furthermore, fin regeneration was also inhibited by the knockdown of raptor, which encodes a component of mTORC1 $[7,8,19]$ using a morpholino antisense oligo nucleotide (MO) (Additional file 4: Figure S4). Taken together, inhibition of mTORC1 signaling with these three inhibitors (rapamycin, Torin1, and AZD8055) or by knockdown of raptor suggests that mTORC1 signaling is required in the pre-blastema formation, blastema formation, and regenerative outgrowth stages during fin regeneration.

We showed that mTORC1 signaling is active in proliferative intra-ray cells and osteoblast progenitors during the pre-blastema formation stage (Figure 2A-F'). To test whether mTORC1 signaling affects cell proliferation before blastema formation, PCNA and Runx2 immunohistochemical staining, a BrdU incorporation assay, and

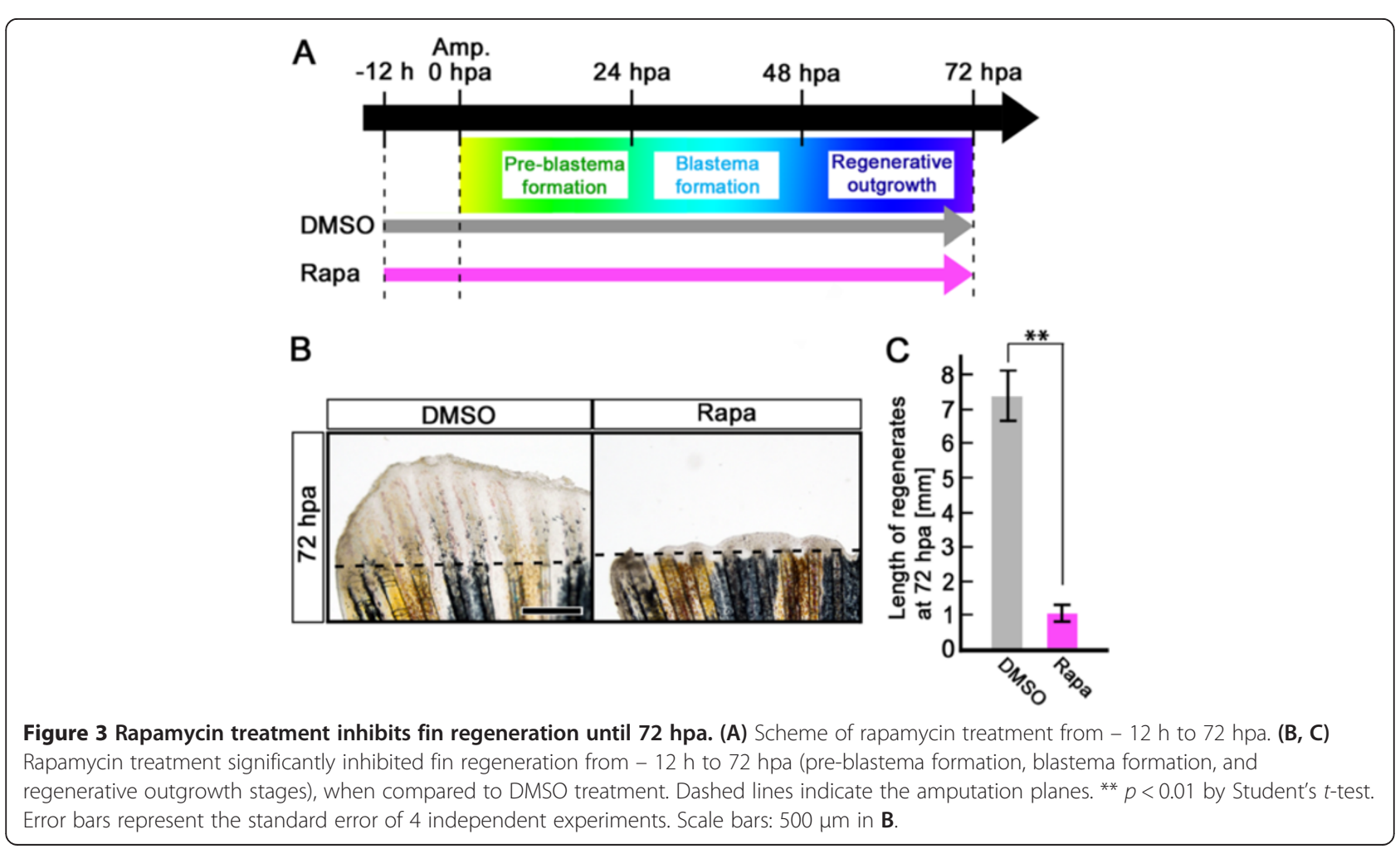


expression of $m s x b$ [20] and the Xenopus ef1- $\alpha$ :EGFP transgene using the transgenic fish XIG8A $[\operatorname{Tg}(e f 1-\alpha$; $E G F P)$ [21] were performed in rapamycin-treated fins. Because cell proliferation starts at approximately $18 \mathrm{hpa}$ in regenerating fins [3], inhibition experiments of mTORC1 signaling by rapamycin treatment were performed from $-12 \mathrm{~h}$ to $18 \mathrm{hpa}$ (or $24 \mathrm{hpa}$ ). mTORC1 signaling inhibition was confirmed by the loss of the p-S6K signal at $24 \mathrm{hpa}$ (Additional file 5: Figure S5). At $18 \mathrm{hpa}$, the number of PCNA-positive cells and the percent of Runx2-positive cells were significantly reduced by rapamycin treatment (Figure 4B-E), whereas the number of apoptotic cells was not increased in the intra-ray and epidermal cells (Figure 4F,G), indicating that inhibition of mTORC1 signaling suppresses cell proliferation without inducing apoptosis. Consistent with PCNA and Runx2 immunohistochemical staining, the number of BrdU incorporated cells in both the intra-ray and epidermis was significantly reduced by the rapamycin treatment at $24 \mathrm{hpa}$ (Figure $4 \mathrm{H}, \mathrm{I})$. It was previously reported that msxb and Xenopus ef1- $\alpha$ :EGFP transgene are molecular markers for mesenchymal progenitor cells [20] and proliferative cells [22] in the regenerating fins, respectively. Similarly to PCNA and Runx2 expression, $m s x b$ and Xenopus ef1- $\alpha$ :EGFP transgene expression was markedly decreased by rapamycin treatment at $24 \mathrm{hpa}$ as determined by whole-mount in situ hybridization and EGFP fluorescence, respectively (Figure 4J,K). These results clearly indicate that $\mathrm{mTORC1}$ signaling is required for cell proliferation, but not in cell survival of intra-ray and epidermal cells before blastema formation.

mTORC1 signaling is required for cell proliferation and cell survival during the regenerative outgrowth stage

Because p-S6K-positive cells start to accumulate underneath the wound epidermis from 24 hpa (Figure 1E), and cell proliferation is suppressed until 24 hpa by mTORC1 signaling inhibition (Figure 4), identifying the function of mTORC1 signaling during blastema formation and regenerative outgrowth is difficult. We next examined the function of mTORC1 signaling during the blastema formation and regenerative outgrowth stages using rapamycin from 24 to $72 \mathrm{hpa}$ (Figure 5A). Regenerative outgrowth was significantly inhibited by rapamycin treatment from 24 to $72 \mathrm{hpa}$ (Figure 5B,C), as observed by rapamycin treatment from $-12 \mathrm{~h}$ to $72 \mathrm{hpa}$ (Figure 3). mTORC1 signaling inhibition was confirmed by the loss of the $\mathrm{p}$-S6K signal at $72 \mathrm{hpa}$ (Additional file 6: Figure S6). In addition, $m s x b$ and connexin 43 (cx43), a molecular marker of proliferating cells [23], expression was downregulated by rapamycin treatment in the regenerative fins (Figure 5D). Consistent with these in situ hybridization results, the number of PCNA-positive cells in both the blastema and epidermis was significantly reduced by rapamycin treatment (Figure 5E,F), as observed before blastema formation (Figure 4). In contrast to the pre-blastema formation stage, the number of apoptotic cells in both the blastema and epidermis was significantly increased by rapamycin treatment during the blastema formation and regenerative outgrowth stages (Figure 5G, $\mathrm{H})$. These results suggest that mTORC1 signaling is required for cell proliferation and cell survival during blastema formation and regenerative outgrowth.

\section{mTORC1 signaling is required for the proliferation and differentiation of bony fin ray after $72 \mathrm{hpa}$}

As shown in Figure 2, mTORC1 signaling was specifically activated in the putative differentiating osteoblasts after 72 hpa. To examine the function of mTORC1 signaling in bony ray formation, the regenerates were treated with rapamycin from 72 to $120 \mathrm{hpa}$. Inhibition of mTORC1 signaling was confirmed by the marked reduction of the $\mathrm{p}$ S6K signal at 120 hpa (Additional file 7: Figure S7). The number of BrdU incorporated cells in the Zns-5-positive osteoblasts was significantly reduced by rapamycin treatment (Figure 6B,C). In addition, the number of Sp7expressing cells and expression domains of col10a1a, which are intermediate markers of skeletogenesis [15], were markedly decreased in the rapamycin-treated fins (Figure 6D,E,F). In contrast to differentiation markers for skeletogenesis, the number of Runx2-positive osteoblast progenitors was unchanged by rapamycin treatment (Figure 6G,H). These results suggest that mTORC1 signaling is required for proliferation and differentiation of the bony fin ray after $72 \mathrm{hpa}$.

\section{mTORC1 signaling does not regulate autophagy in fin regeneration}

A recent study revealed that autophagy is required for zebrafish fin regeneration under the control of MAPK/Erk signaling pathway [24]. Because the mTORC1 signaling pathway is known to inhibit autophagy $[7,8]$, we examined whether autophagy was affected by inhibition of mTORC1 signaling during fin regeneration. As determined using a GFP-microtubule-associated protein 1 light chain 3 isoform (GFP-LC3) transgenic line, autophagy was markedly upregulated from 1 to 4 days post amputation (dpa) [24]. Using an LC3B antibody, we detected LC3 in the wound epidermis at $24 \mathrm{hpa}$, and by $72 \mathrm{hpa}$, LC3 localization in the wound epidermis was maintained (Additional file 8: Figure S8). Moreover, LC3 protein level and localization were not affected by rapamycin treatment (Additional file 8: Figure S8), suggesting that the mTORC1 signaling pathway does not regulate fin regeneration via autophagy.

\section{IGF-1 receptor (IGF-1R)/PI3K and Wnt signaling pathways regulate $\mathrm{mTORC} 1 / \mathrm{S} 6 \mathrm{~K}$ in fin regeneration}

It is well known that the mTORC1/S6K pathway is regulated through the receptor tyrosine kinase/PI3K/Akt 


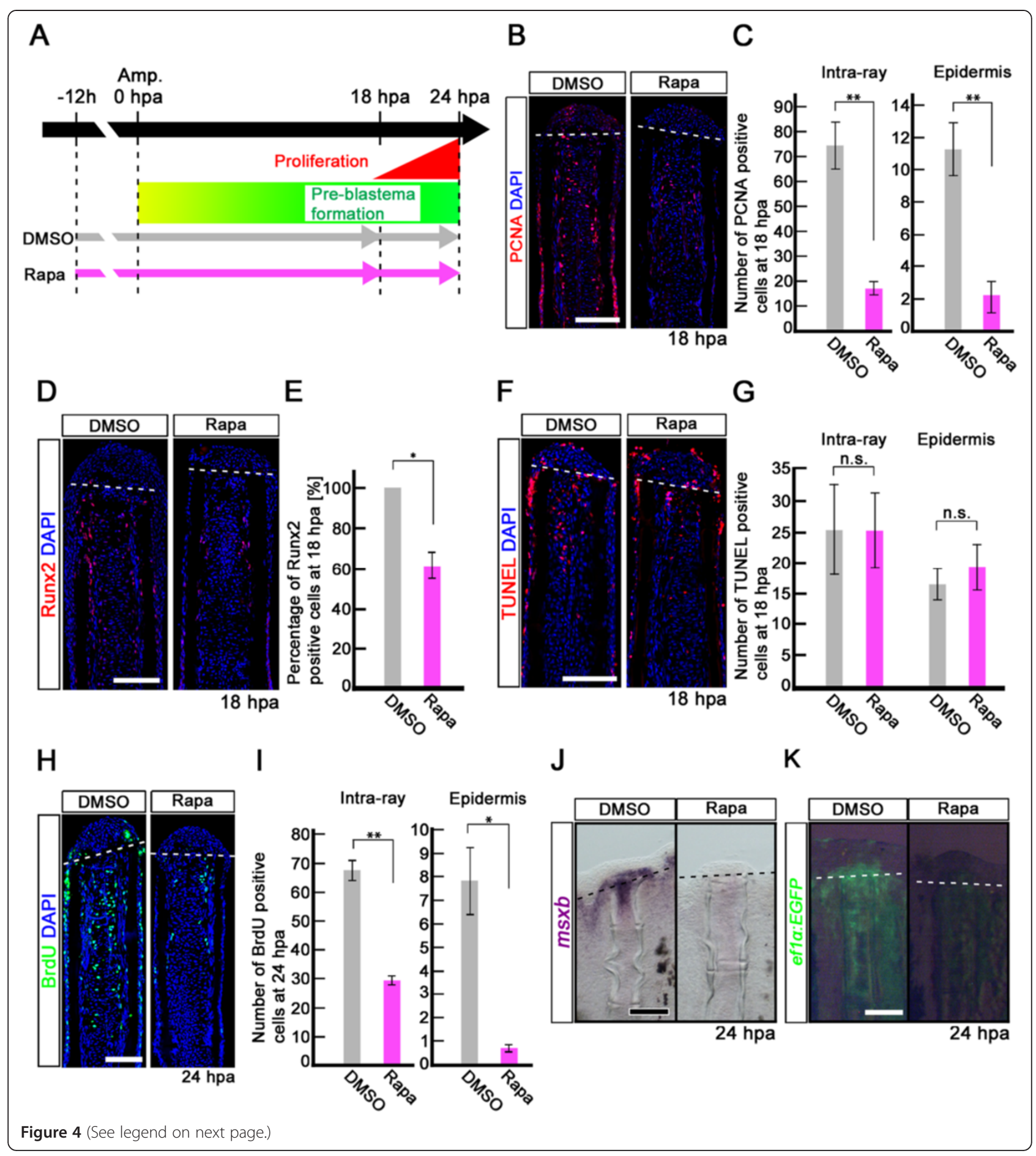


(See figure on previous page.)

Figure 4 Rapamycin treatment inhibits proliferation of intra-ray and epidermal cells, but not apoptosis before blastema formation.

(A) Scheme of rapamycin treatment before blastema formation. (B, C) PCNA-stained fin sections and quantification of PCNA-positive cells in the intra-ray and epidermis at $18 \mathrm{hpa}$. The number of PCNA-positive cells was significantly reduced by rapamycin treatment in both the intra-ray and epidermis at $18 \mathrm{hpa} .{ }^{* *} p<0.01$ by Student's $t$-test. Error bars represent the standard error of 5 independent experiments. Scale bars: $100 \mu \mathrm{m}$. (D,E) Runx2-stained fin sections and quantification of Runx2-positive cells in the intra-ray. Rapamycin treatment significantly reduced the percentage of Runx2-positive cells at $18 \mathrm{hpa} .{ }^{*} p<0.05$ by Student's $t$-test. Error bars represent the standard error of 3 independent experiments. Scale bars: $100 \mu \mathrm{m}$. $(\mathbf{F}, \mathbf{G})$ TUNEL-stained fin sections and quantification of TUNEL-positive intra-ray and epidermal cells. Cell death was not increased in both the intra-ray and epidermis at $18 \mathrm{hpa}$. Error bars represent the standard error of 6 independent experiments. Scale bars: 100 Hm. (H, I) BrdU-stained fin sections and quantification of BrdU-positive cells in the intra-ray and epidermis. Rapamycin treatment significantly reduced the number of BrdU-positive cells in the intra-ray and epidermis at 24 hpa. ${ }^{*} p<0.05,{ }^{* *} p<0.01$ by Student's $t$-test. Error bars represent the standard error of 3 independent experiments. Scale bars: $100 \mu \mathrm{m}$. DAPI fluorescent signal (blue) indicates the presence of nuclei (B, D, F, H). Dashed white lines indicate the amputation planes (B, D, F, H). (J) Expression of $m s x b$ was examined by in situ hybridization at $24 \mathrm{hpa}(\mathrm{n}=3)$. The $m s x b$ expression was barely detectable in rapamycin-treated fin regenerates. Scale bars: $200 \mu \mathrm{m}$. (K) EGFP fluorescence of Tg(ef1-a;EGFP) fin regenerates at $24 \mathrm{hpa}(\mathrm{n}=3)$. The EGFP fluorescence was lost in rapamycintreated fin regenerates. Scale bars: $200 \mu \mathrm{m}$. Dashed lines indicate the amputation plane (J, K).

pathway in cell proliferation and metabolism $[7,8]$. To examine the upstream signaling pathway regulating mTORC1/S6K signaling in fin regeneration, various inhibitors for IGF, Wnt, Fgf, and ROS signaling pathways were tested from $-12 \mathrm{~h}$ to $24 \mathrm{hpa}$ or from 24 to $48 \mathrm{hpa}$, and S6K activation was determined; IGF signaling (LY294002 [25]: a PI3K inhibitor, NVP-ADW742 [26]: a IGF-1 receptor kinase inhibitor), Wnt signaling (IWP-2 [27]: a Wnt/ $\beta$ catenin signaling inhibitor), Fgf signaling (SU5402 [28]: a Fgf receptor1 inhibitor), MAPK/Erk signaling (U0126 [29]: a MAPK/Erk inhibitor), and ROS signaling (VAS2870 [30]: an inhibitor of NADPH oxidase). No inhibitory effect for S6K activation was observed using SU5402, U0126, or VAS2870, even though fin regeneration was suppressed (Additional file 9: Figure S9). However, LY294002, NVPADW742, and IWP-2 inhibitor treatment markedly reduced the p-S6K signal in blastema and epidermal cells. The inhibitory effect of LY294002, NVP-ADW742, and IWP-2 on S6K activation was nearly similar to that of rapamycin during fin regeneration (Figure 7). These results suggest that both IGF-1R/PI3K and Wnt pathways activate mTORC1 in blastema and epidermal cells during zebrafish caudal fin regeneration.

\section{Discussion}

Based on the localization of $\mathrm{p}-\mathrm{S} 6 \mathrm{~K}$, we show the spatiotemporal distributions of cells in which mTORC1 signaling is activated during fin regeneration (Figure 1). Weak p-S6K fluorescent signals were observed in the intra-ray cells and epidermis as early as 6 hpa. Further, the p-S6Kpositive cells were found to be distributed in locations proximal to the amputation plane by $36 \mathrm{hpa}$, indicating that $\mathrm{mTORC} 1$ is one of the earliest signaling in fin regeneration, which is reminiscent of other signaling pathways such as Wnt, Fgf, retinoic acid, and Activin [2,9,10]. We also show that $\mathrm{mTORC} 1$ signaling is active in proliferative blastema cells, wound epidermal cells, and osteoblasts during blastema formation and regenerative outgrowth (after $24 \mathrm{hpa}$ ) (Figure 2). Inhibition of mTORC1 signaling from 24 to 72 hpa suppresses proliferation of these cells through the inhibition of S6K activation (Figure 5, Additional file 6: Figure S6). These results suggest that mTORC1 signaling is continuously required for cellular proliferation during the blastema formation and regenerative outgrowth stages via protein synthesis.

It is well established that mTORC1 signaling activates anabolic processes, including the synthesis of proteins, nucleotides, and lipids, and, as a result, these processes promote cell growth and proliferation [7,8,31]. Protein and nucleotide syntheses are controlled through the activation of S6K, and lipid synthesis is promoted by activating sterol regulatory element binding proteins in an S6K-dependent or -independent manner [8,31]. Based on these findings, the p-S6K-positive cells observed in regenerating fins appear to be in a higher metabolic state that is stimulated by a wound signal. It would be significant to isolate and analyze these cells under high metabolic conditions during fin regeneration.

A recent paper demonstrated that zebrafish caudal fin regeneration is slightly inhibited by rapamycin treatment from 0 to $35 \mathrm{dpa}$ [32]. However, the inhibitory effect of rapamycin observed was milder than the effect observed in our study. The rapamycin concentration used in this report was lower than that used in the present study $(50 \mathrm{nM}$ in a previous report versus $2.4 \mu \mathrm{M}$ in the present study), which may explain this discrepancy. In addition, while fish were pretreated with rapamycin before amputation $(-12 \mathrm{~h})$ in our experiments, no pretreatment was performed in the previous report. In fact, we found that at least $6 \mathrm{~h}$ of pretreatment is needed to observe the loss of $\mathrm{p}$-S6K immunostaining. When fish were treated with rapamycin from $0 \mathrm{hpa}$, the inhibitory effect of rapamycin on fin regeneration was markedly reduced, even when using a $2.4 \mu \mathrm{M}$ concentration of rapamycin (data not shown). It is likely that these two different experimental conditions may be responsible for the difference observed between our study and the previous report.

A previous report showed that insulin-like growth factor $2 b(i g f 2 b)$, which is expressed in blastema, activates the IGF 


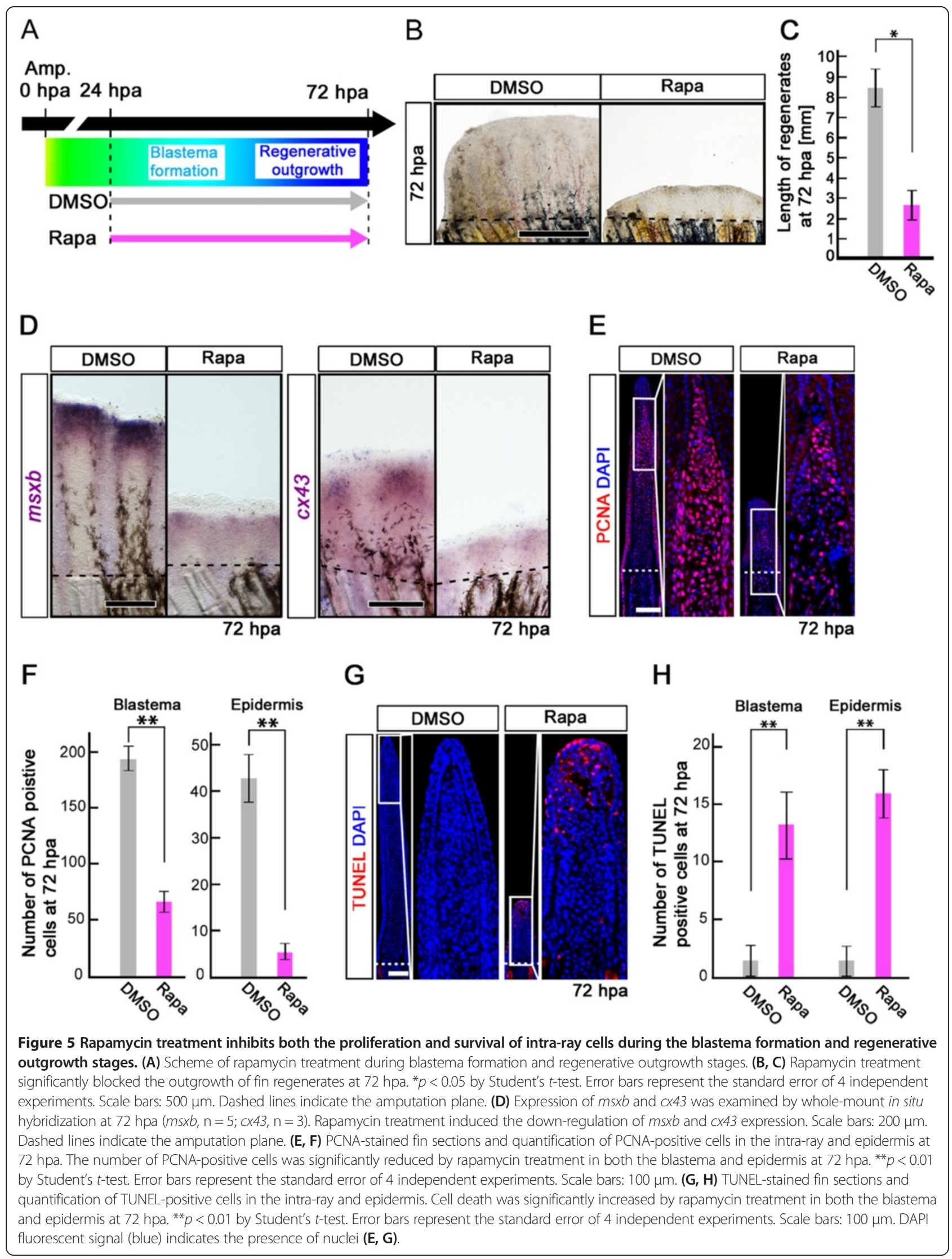




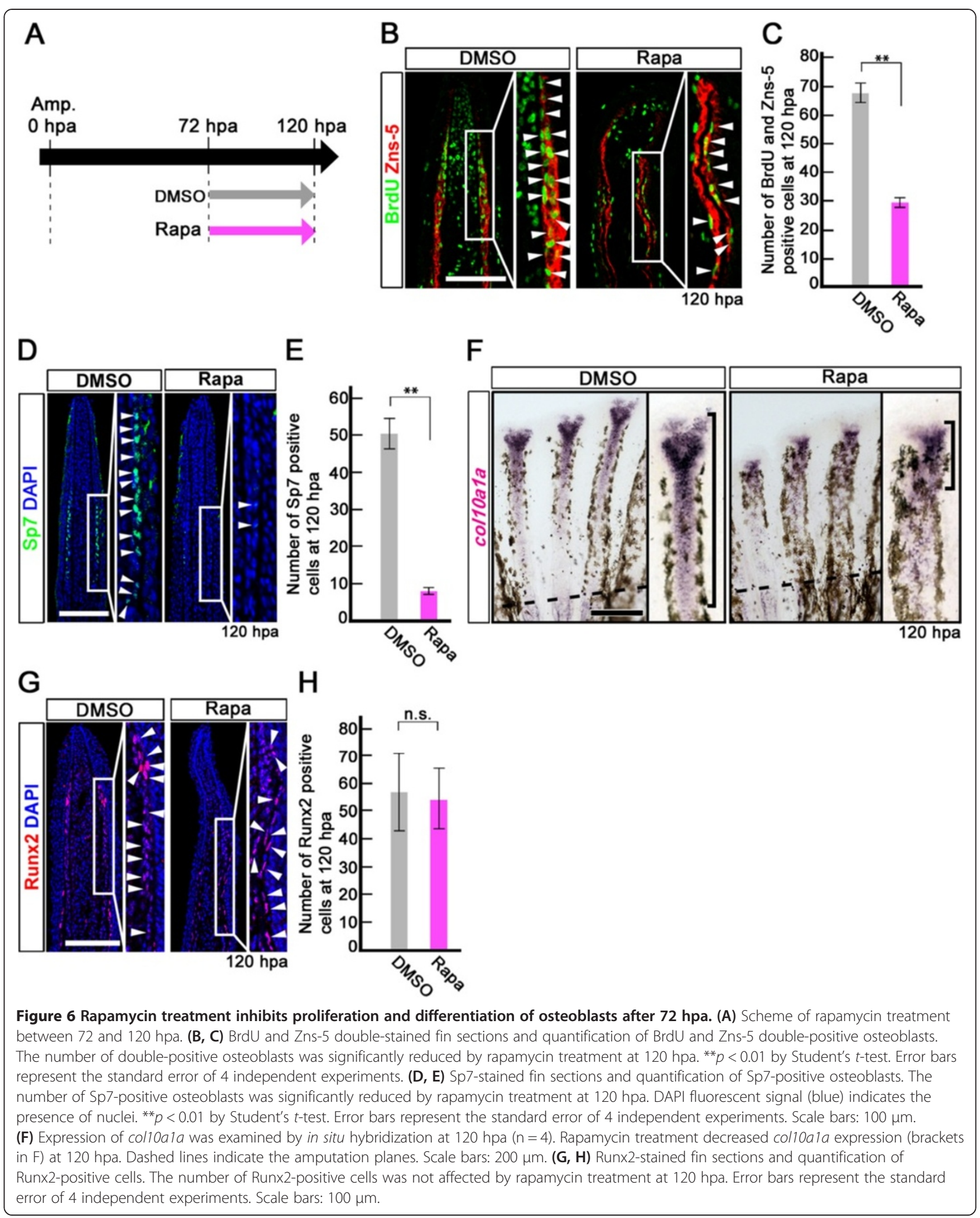




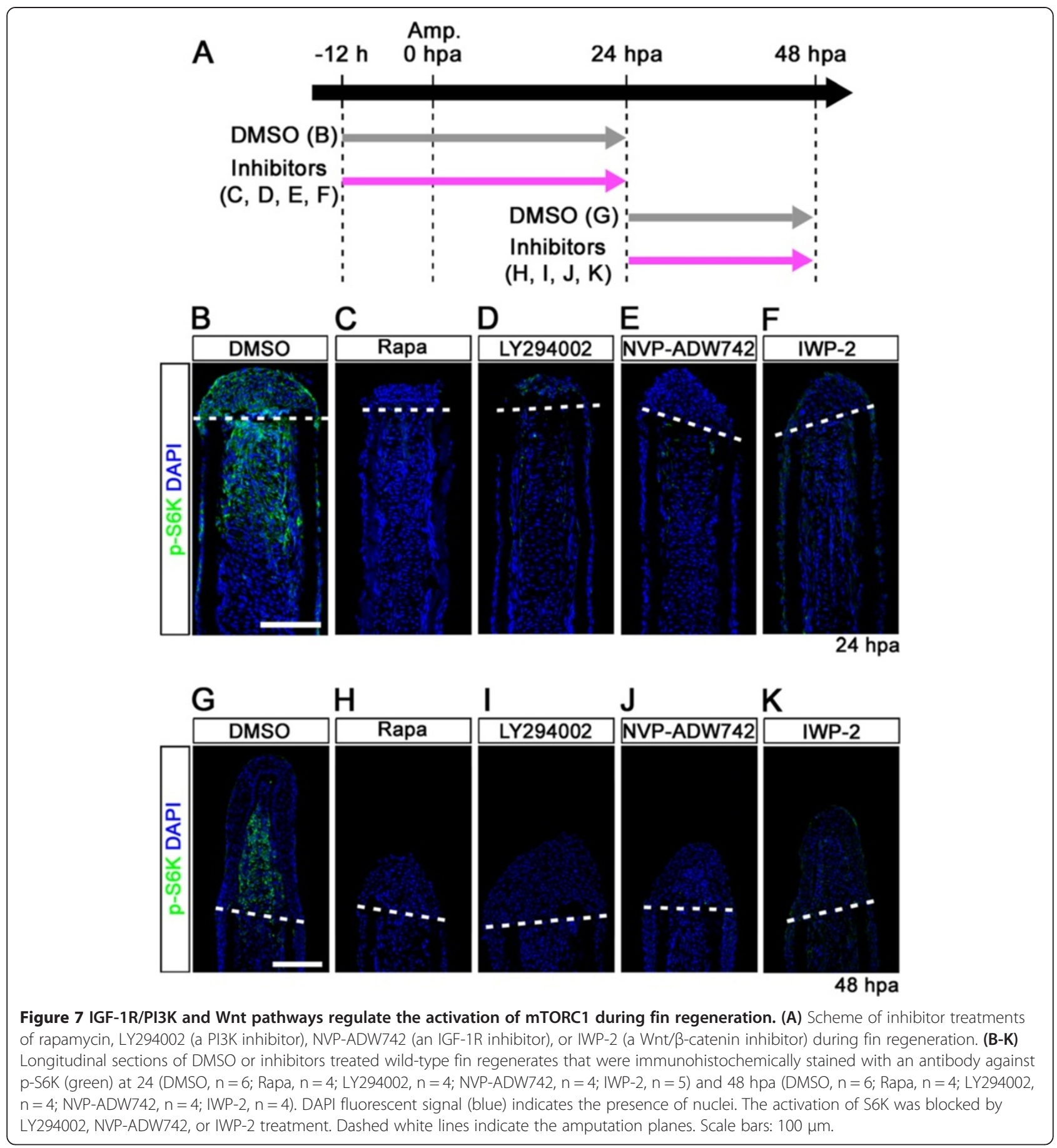

signaling pathway in the apical epithelium cells through two receptors, insulin-like growth factor 1a receptor (Igf1ra) and Igf1rb [33]. Pharmacological inhibition using NVPADW541 or NVP-ADW742 caused apoptosis in the wound epidermis and blocked blastema formation, indicating that IGF signaling between blastema and wound epidermis is critical for cell survival in the wound epidermis and cell proliferation in the blastema [33]. These results are consistent with some of our results showing that mTORC1 signaling is active in the wound epidermis before blastema formation, and is regulated by the IGF-1R/PI3K signaling pathway. However, two phenotypic differences were observed between mTORC1 and IGF-1R inhibition during fin regeneration. One is that cell death was significantly increased in the wound epidermis by IGF-1R inhibition at 24 hpa [33], but not by rapamycin treatment from -12 h to 18 hpa (Figure 4). Because the IGF signaling is one of upstream signaling leading to mTORC1/S6K pathway, the other 
signaling pathways might be involved in the wound epidermis survival. The second is that activation of mTORC1 based on p-S6K localization was observed not only in the wound epidermis, but also in intra-ray cells as early as 6 hpa. It is possible that, in intra-ray cells, mTORC1 signaling is activated through IGF-1Rs, other than Igf1ra and Igflrb.

In addition to IGF-1R/PI3K signaling, we also found that Wnt signaling controls mTORC1 activation in both blastema and epidermal cells during fin regeneration by using IWP-2 (Figure 7). It has already been shown that Wnt signaling directly activates mTORC1 through the inhibition of glycogen synthase kinase 3 and tuberous sclerosis $1 / 2$ in zebrafish and mice [34], as well as in mammalian cell lines [35]. In zebrafish caudal fin regeneration, Wnt/ $\beta$-catenin signaling indirectly regulates the proliferation of blastema cells, patterning of epidermis, and differentiation and patterning of bone, mediated through many signaling pathways such as Activin, Notch, Fgf, retinoic acid, Hedgehog, IGF, and Bmp [10]. Because mTORC1 signaling could be mediated directly or indirectly through IGF signaling regulated by Wnt/ $\beta$ catenin signaling, further detailed investigations are needed to elucidate the hierarchical relationship and crosstalk between IGF-1R/PI3K/mTORC1 and other signaling pathways downstream of $\mathrm{Wnt} / \beta$-catenin during fin regeneration.

\section{Conclusions}

In this study, we showed that the mTORC1 signaling is activated in intra-ray and wound epidermal cells as early as $6 \mathrm{hpa}$, and continues to be activated in proliferative blastema cells, wound epidermal cells, and osteoblasts during fin regeneration. Inhibition of $\mathrm{mTORC} 1$ signaling during the pre-blastema formation, blastema formation, and regenerative outgrowth stages by rapamycin suggests that mTORC1 signaling is required for fin regeneration. Before blastema formation, mTORC1 signaling is required for intra-ray and wound epidermal cell proliferation, but not in cell survival, whereas during the blastema formation and regenerative outgrowth stages, mTORC1 signaling is required for both blastema and wound epidermal cell proliferation and survival. Furthermore, during the regenerative outgrowth stage, $\mathrm{mTORC} 1$ signaling is required for both proliferation and differentiation of the bony fin ray. In summary, our study is the first report that uncovers the requirement and significance of mTORC1 signaling for the cell proliferation, cell survival, or differentiation of intra-ray cells, blastema cells, wound epidermal cells, and/ or osteoblasts during vertebrate epimorphic regeneration downstream of IGF and Wnt signaling pathways.

\section{Methods}

Zebrafish husbandry, fin amputation, drug treatments, and in vivo electroporation

All zebrafish experiments were performed under ethical approval of the Hiroshima University Animal Research
Committee (Permit Number: F13-1). Maintenance and caudal fin amputation of adult zebrafish (AB/Tüebingen strain) were performed as described previously [36]. Transgenic zebrafish XIG8A [Tg(ef1- $\alpha: E G F P)]$ [21] was used for fin amputation experiments.

During drug treatments, fish were kept in fish water at $28.5^{\circ} \mathrm{C}$ and the fish water with drug was replaced daily. 2.4 $\mu \mathrm{M}$ rapamycin (LC Laboratories), $100 \mathrm{nM}$ Torin1 (Calbiochem), 1.2 $\mu \mathrm{M}$ AZD8055 (AdooQ Biosciences), $10 \mu \mathrm{M}$ LY294002 (Calbiochem), $5 \mu \mathrm{M}$ NVP-ADW742 (AdooQ Biosciences), $14 \mu \mathrm{M}$ SU5402 (Calbiochem), $10 \mu \mathrm{M}$ IWP-2 (Promega), $25 \mu \mathrm{M}$ U0126 (Promega), and $1 \mu \mathrm{M}$ VAS2870 (Enzo Life Sciences) were used as specific inhibitors. All were dissolved in DMSO and final DMSO concentration in fish water was $0.1 \%$, except SU5402 (0.17\%). The control fish were kept in fish water with $0.1 \%$ DMSO.

For MO knockdown experiments, in vivo electroporation was performed as described previously [37]. The MO was micro-injected between each bony fin ray and electroporated before fin amputation. We used fluorescent tagged-MO targeted against raptor and 5-base mismatch control MO (Gene Tools, Inc.) as following; a raptor MO (5'-ATGGATGGATGGATGCTCACCTATC-3') [19], a 5-mismatch control MO (5' - ATGaATGaATGaATGaTCACaTATC $-3^{\prime}$, lower case letters indicate mismatch pairs).

\section{Immunohistochemical staining and whole-mount in situ hybridization}

The amputated fins were fixed in $4 \%$ paraformaldehyde in $0.1 \mathrm{M}$ phosphate-buffered saline (PBS) overnight at $4^{\circ} \mathrm{C}$. After fixation, the fins were treated with PBS containing $30 \%$ sucrose overnight, and were embedded with TissueTek O.C.T. compound (Sakura Finetek). The embedded fins were frozen and sectioned to $14 \mu \mathrm{m}$ thickness by using a Leica CM3050S. The sections were dehydrated and rehydrated through a methanol/PBS-0.1\% Tween series for $5 \mathrm{~min}$ each. After rehydration, the sections were blocked with PBST (PBS and 0.1\% Tween20) containing 5\% sheep serum for $3 \mathrm{~h}$ at room temperature and then incubated in PBST with primary antibody/antibodies overnight at $4^{\circ} \mathrm{C}$. The following primary antibodies were used: anti-PCNA mouse monoclonal antibody at 1:1000 (Sigma, \#P8825) [36]; anti-BrdU rat monoclonal antibody at 1:100 (Abcam, ab6326); phospho-S6 ribosomal protein (Ser240/244) rabbit polyclonal antibody at 1:300 (Cell Signaling, \#2215) [38]; anti-Runx2 mouse monoclonal antibody at 1:100 (Sant Cruz Biotechnology, 27-K) [10,11]; anti-Zns-5 mouse monoclonal antibody at 1:300 (a kindly gift from Dr. Ishitani) [14]; anti-Sp7 rabbit polyclonal antibody at 1:1000 (Santa cruz, sc-22536-R) [11]; anti-LC3B rabbit polyclonal antibody at 1:300 (Abcam, ab51520) [39]. The following secondary antibodies were used: Alexa Fluor ${ }^{\circ}$ 
488 goat anti-rabbit IgG antibody at 1:500 (Invitrogen, Life Technologies Corp.); Alexa Fluor ${ }^{\circ} 488$ goat anti-rat IgG antibody at 1:500 (Invitrogen, Life Technologies Corp.); Alexa Fluor 594 goat anti-mouse IgG antibody at 1:500 (Invitrogen, Life Technologies Corp.); Alexa Fluor 594 goat anti-rabbit IgG Antibody at 1:500 (Invitrogen, Life Technologies Corp.). 4',6-diamidino-2-phenylindole (DAPI) was used for nuclei staining at a concentration of 1:1000. The images were captured using an Olympus FV1000-D confocal microscope with the same exposure times using the FluoView software.

Whole-mount in situ hybridization analyses were performed as described previously [40] with 60 minute proteinase $\mathrm{K}$ treatment.

\section{BrdU incorporation assays and cell death detection}

BrdU incorporation assays were performed as described previously [36]. Fin-amputated fish were allowed to regenerate in the fish water containing with $50 \mu \mathrm{g} / \mathrm{ml} \mathrm{BrdU} \mathrm{be-}$ tween 18 to $24 \mathrm{hpa}$ or 108 to $120 \mathrm{hpa}$. After incubation, the regenerating fins were cut and BrdU-labeled cells were detected as described previously [36]. For detection of apoptotic cells, we performed TUNEL staining using an In Situ Cell Death Detection Kit (Roche Applied Science) according to the manufacture's instruction.

\section{Additional files}

Additional file 1: Figure S1. Treatment of Torin1 or AZD8055 inhibits fin regeneration until $72 \mathrm{hpa}$. (A) Scheme of Torin1 or AZD8055 treatment from $-12 \mathrm{~h}$ to $72 \mathrm{hpa}$. $(\mathrm{B}, \mathrm{C})$ Treatment of both inhibitors significantly inhibited fin regeneration from $-12 \mathrm{~h}$ to $72 \mathrm{hpa}$ (pre-blastema formation, blastema formation, and regenerative outgrowth stages), when compared to DMSO treatment. Dashed lines indicate the amputation planes. ${ }^{*} p<0.05,{ }^{*} p<0.01$ by Student's $t$-test. Error bars represent the standard error of 4 independent experiments. Scale bars: $500 \mu \mathrm{m}$ in B.

Additional file 2: Figure S2. Distributions of $\mathrm{p}-\mathrm{S} 6 \mathrm{~K}$ in rapamycin, Torin1, or AZD8055-treated fin regenerates at $72 \mathrm{hpa}$. (A) Scheme of rapamycin, Torin1, or AZD8055 treatment from - $12 \mathrm{~h}$ to $72 \mathrm{hpa}$. (B) Longitudinal sections of wild-type fin regenerates that were immunohistochemically stained with an antibody against p-S6K (green) at $72 \mathrm{hpa}$ (DMSO, $\mathrm{n}=5$; Rapa, $\mathrm{n}=3$; Torin1, $\mathrm{n}=3$; AZD8055, $\mathrm{n}=3$ ). DAPI fluorescent signal (blue) indicates the presence of nuclei. At $72 \mathrm{hpa}$, the p-S6K fluorescent signal was lost in rapamycin, Torin1, or AZD8055-treated fin regenerates. Dashed white lines indicate the amputation plane. Scale bars: $100 \mu \mathrm{m}$.

Additional file 3: Figure S3. $p-S 6 K$ signals were markedly reduced by 3 or $6 \mathrm{~h}$ treatment with inhibitors (rapamycin, Torin1, and AZD8055). (A) Scheme of rapamycin, Torin1, or AZD8055 treatment from $24 \mathrm{~h}$ to 27 or 30 hpa. (B-I) Longitudinal sections of wild-type fin regenerates that were immunohistochemically stained with an antibody against $\mathrm{p}-\mathrm{S6K}$ (green) at 27 hpa (DMSO, $n=3$; Rapa, $n=3$; Torin 1, $n=3$; AZD8055, $n=3$ ) or $30 \mathrm{hpa}$ (DMSO, $n=3$; Rapa, $n=3$; Torin1, $n=3$; AZD8055, $n=3$ ). DAPI fluorescent signal (blue) indicates the presence of nuclei. The p-S6K signals were markedly reduced by 3 or $6 \mathrm{~h}$ treatment with inhibitors (rapamycin, Torin 1 , and AZD8055). Dashed white lines indicate the amputation plane. Scale bars: $100 \mu \mathrm{m}$.

Additional file 4: Figure S4. Fin regeneration and activation of mTORC1 signaling are inhibited by raptor knock-down. (A,B) Wild-type fin electroporated in the ventral half with fluorescein-labeled MOs at $72 \mathrm{hpa}$.
Fin regeneration was significantly inhibited by raptor knock-down. Dashed lines indicate the amputation planes. Scale bars: $500 \mu \mathrm{m}$. ${ }^{* *} p<0.01$ by Student's $t$-test. Error bars represent the standard error of 5 independent experiments. (C) Longitudinal sections of wild-type fin regenerates that were immunostained with antibodies against $\mathrm{p}-\mathrm{S} 6 \mathrm{~K}$ (red) at $24 \mathrm{hpa}$ (5-mismached raptor $\mathrm{MO}, \mathrm{n}=5$; raptor $\mathrm{MO}, \mathrm{n}=5$ ). Green fluorescence indicates the presence of $\mathrm{MO}$ in the electroporated cells. Merged views showed that cells incorporated 5-mismached raptor MO, but not raptor MO, are p-S6K-positive (arrowheads). It should be noted that fluorescent signals of 5-mismached raptor MO and p-S6K overlap only in the cytosol, because p-S6K are localized in the cytosol, not in the nucleus. Dashed white lines indicate the amputation planes. Scale bars: $100 \mu \mathrm{m}$.

Additional file 5: Figure S5. Distributions of $\mathrm{p}-\mathrm{S} 6 \mathrm{~K}$ in rapamycin-treated fin regenerates at $24 \mathrm{hpa}$. (A) Scheme of rapamycin treatment from $-12 \mathrm{~h}$ to $24 \mathrm{hpa}$. (B) Longitudinal sections of wild-type fin regenerates that were immunohistochemically stained with an antibody against p-S6K (green) at $24 \mathrm{hpa}$ (DMSO, $n=4$; Rapa, $n=4$ ). DAPI fluorescent signal (blue) indicates the presence of nuclei. At $24 \mathrm{hpa}$, the p-S6K fluorescent signal was lost in rapamycin-treated fin regenerates. Dashed white lines indicate the amputation plane. Scale bars: $100 \mu \mathrm{m}$.

Additional file 6: Figure S6. Distributions of p-S6K in fin regenerates treated with rapamycin from 24 to $72 \mathrm{hpa}$. (A) Scheme of rapamycin treatment from 24 to $72 \mathrm{hpa}$. (B) Longitudinal sections of wild-type fin regenerates that were immunohistochemically stained with an antibody against p-S6K (green) at $72 \mathrm{hpa}(\mathrm{DMSO}, \mathrm{n}=5$; Rapa, $\mathrm{n}=5$ ). DAPI fluorescent signal (blue) indicates the presence of nuclei. At $72 \mathrm{hpa}$, the p-S6K fluorescent signal was lost in rapamycin-treated fin regenerates. Dashed white lines indicate the amputation plane. Scale bars: $100 \mu \mathrm{m}$.

Additional file 7: Figure S7. Distributions of $\mathrm{p}-\mathrm{S} 6 \mathrm{~K}$ in fin regenerates treated with rapamycin from 72 to $120 \mathrm{hpa}$. (A) Scheme of rapamycin treatment from 72 to $120 \mathrm{hpa}$. (B) Longitudinal sections of wild-type fin regenerates that were immunohistochemically stained with an antibody against $\mathrm{p}$-S6K (green) at $120 \mathrm{hpa}$ (DMSO, $n=3 ;$ Rapa, $n=3$ ). DAPI fluorescent signal (blue) indicates the presence of nuclei. At $120 \mathrm{hpa}$, the fluorescent signal of $\mathrm{p}-\mathrm{S} 6 \mathrm{~K}$ was markedly reduced by rapamycin treatment. Scale bars: $100 \mu \mathrm{m}$.

Additional file 8: Figure S8. Distributions of LC3B in rapamycin treated fin regenerates. (A) Scheme of rapamycin treatment from -12 to $24 \mathrm{hpa}$ or from 36 to $72 \mathrm{hpa}$. $(B, C)$ Longitudinal sections of DMSO or rapamycin treated wild-type fin regenerates that were immunohistochemically stained with an antibody against LC3B (green) at $24 \mathrm{hpa}$ (DMSO, $\mathrm{n}=4$; Rapa, $\mathrm{n}=4$ ) and $72 \mathrm{hpa}$ (DMSO, $\mathrm{n}=3$; Rapa, $\mathrm{n}=3$ ). DAPI fluorescent signal (blue) indicates the presence of nuclei. LC $3 B$ is specifically localized in the wound epidermis at 24 and $72 \mathrm{hpa}$. Dashed white lines indicate the amputation planes. Scale bars: $100 \mu \mathrm{m}$.

Additional file 9: Figure S9. Fgf, MAPK/Erk, and ROS signaling pathways do not regulate the activation of mTORC1 during fin regeneration. (A) Scheme of inhibitor treatments of SU5402 (a Fgf receptor1 inhibitor), U0126 (a MAPK/Erk inhibitor), and VAS2870 (an NADPH oxidase inhibitor: ROS signaling) during fin regeneration. (B-I) Longitudinal sections of DMSO or inhibitors treated wild-type fin regenerates that were immunohistochemically stained with an antibody against p-S6K (green) at 24 or $48 \mathrm{hpa}$. DAPI fluorescent signal (blue) indicates the presence of nuclei. The p-S6K signals in SU5402-, U0126-, and VAS2870-treated fins were approximately comparable to those in DMSO-treated fins at both $24 \mathrm{hpa}$ (DMSO, $n=6 ;$; SU5402, $n=5$; U0126, $n=3 ;$ VAS2870, $n=3$ ) and $48 \mathrm{hpa}$ (DMSO, $n=6 ;$ SU5402, $n=4$; U0126, $n=3$; VAS2870, $n=3$ ), even though these inhibitors, at $48 \mathrm{hpa}$, prevented fin regeneration. Dashed white lines indicate the amputation planes. Scale bars: $100 \mu \mathrm{m}$.

\section{Abbreviations}

BrdU: 5-bromo-2'-deoxyuridine; DAPI: 4',6-diamidino-2-phenylindole; DMSO: Dimethyl sulfoxide; EGFP: Enhanced green fluorescent protein; qRT-PCR: quantitative real-time polymerase chain reaction.

\section{Competing interests}

The authors declare that they have no competing interests. 


\section{Authors' contributions}

$\mathrm{KH}$ and $\mathrm{YK}$ designed experiments. $\mathrm{KH}, \mathrm{TS}$, and $\mathrm{SH}$ performed the experiments. $\mathrm{KH}$ and $\mathrm{YK}$ wrote the manuscript. All the authors discussed the results and commented on the manuscript. All authors read and approved the final manuscript.

\section{Acknowledgements}

We thank Institute for Amphibian Biology in Hiroshima University for cryostat sectioning; Natural Science Center for Basic Research and Development in

Hiroshima University for electroporation; Graduate School of Integrated Arts and Sciences in Hiroshima University for cryostat sectioning; Dr. Koichi Kawakami for providing the transgenic fish XIG8A; Dr. Tohru Ishitani for providing Zns-5 antibody. We also thank the members of Kikuchi and Atsushi Suzuki laboratories in Hiroshima University for helpful discussion and critical comments. This study was supported by grants from Grant-in-Aid for Scientific Research from the JSPS (KAKENHI 23616002) to Y.K., and from Hiroshima University Alumni Association Research Grant \& Hiroshima University Support Foundation Research and Grant-in-Aid for Scientific Research from the JSPS (KAKENHI 26 - 6771) to K.H.

Received: 10 July 2014 Accepted: 25 November 2014

Published online: 06 December 2014

\section{References}

1. Tanaka EM, Reddien PW: The cellular basis for animal regeneration. Dev Cell 2011, 21(1):172-185.

2. Gemberling M, Bailey TJ, Hyde DR, Poss KD: The zebrafish as a model for complex tissue regeneration. Trends Genet 2013, 29(11):611-620.

3. Nechiporuk A, Keating MT: A proliferation gradient between proximal and msxb-expressing distal blastema directs zebrafish fin regeneration. Development 2002, 129(11):2607-2617.

4. Akimenko MA, Marí-Beffa M, Becerra J, Géraudie J: Old questions, new tools, and some answers to the mystery of fin regeneration. Dev Dyn 2003, 226(2):190-201.

5. Poss KD, Keating MT, Nechiporuk A: Tales of regeneration in zebrafish. Dev Dyn 2003, 226(2):202-210.

6. Grotek B, Wehner D, Weidinger G: Notch signaling coordinates cellular proliferation with differentiation during zebrafish fin regeneration. Development 2013, 140(7):1412-1423.

7. Li J, Kim SG, Blenis J: Rapamycin: One Drug. Many Effects. Cell Metab 2014, 19(3):373-379.

8. Laplante M, Sabatini DM: mTOR signaling in growth control and disease. Cell 2012, 149(2):274-293.

9. Tal TL, Franzosa JA, Tanguay RL: Molecular signaling networks that choreograph epimorphic fin regeneration in zebrafish - a mini-review. Gerontology 2010, 56(2):231-240.

10. Wehner D, Cizelsky W, Vasudevaro MD, Ozhan G, Haase C, KagermeierSchenk B, Röder A, Dorsky RI, Moro E, Argenton F, Kühl M, Weidinger G: Wnt/ $\beta$-Catenin Signaling Defines Organizing Centers that Orchestrate Growth and Differentiation of the Regenerating Zebrafish Caudal Fin. Cell Rep 2014, 6(3):467-481.

11. Stewart S, Gomez AW, Armstrong BE, Henner A, Stankunas K: Sequential and opposing activities of Wnt and BMP coordinate zebrafish bone regeneration. Cell Rep 2014, 6(3):482-498.

12. Gauron C, Rampon C, Bouzaffour M, Ipendey E, Teillon J, Volovitch M, Vriz S: Sustained production of ROS triggers compensatory proliferation and is required for regeneration to proceed. Sci Rep 2013, 3:2084.

13. Brown AM, Fisher S, lovine MK: Osteoblast maturation occurs in overlapping proximal-distal compartments during fin regeneration in zebrafish. Dev Dyn 2009, 238(11):2922-2928.

14. Woods AL, Hall PA, Shepherd NA, Hanby AM, Waseem NH, Lane DP, Levison DA: The assessment of proliferating cell nuclear antigen (PCNA) immunostaining in primary gastrointestinal lymphomas and its relationship to histological grade, $\mathrm{S}+\mathrm{G} 2+\mathrm{M}$ phase fraction (flow cytometric analysis) and prognosis. Histopathology 1991, 19(1):21-27.

15. Johnson SL, Weston JA: Temperature-sensitive mutations that cause stage-specific defects in Zebrafish fin regeneration. Genetics 1995 , 141(4):1583-1595.

16. Li N, Felber K, Elks P, Croucher P, Roehl HH: Tracking gene expression during zebrafish osteoblast differentiation. Dev Dyn 2009, 238(2):459-466.

17. Thoreen CC, Kang SA, Chang JW, Liu Q, Zhang J, Gao Y, Reichling LJ, Sim T, Sabatini DM, Gray NS: An ATP-competitive mammalian target of rapamycin inhibitor reveals rapamycin-resistant functions of mTORC1. J Biol Chem 2009, 284(12):8023-8032.

18. Chresta CM, Davies BR, Hickson I, Harding T, Cosulich S, Critchlow SE, Vincent JP, Ellston R, Jones D, Sini P, James D, Howard Z, Dudley P, Hughes G, Smith L, Maguire S, Hummersone M, Malagu K, Menear K, Jenkins R, Jacobsen M, Smith GC, Guichard S, Pass M: AZD8055 is a potent, selective, and orally bioavailable ATP-competitive mammalian target of rapamycin kinase inhibitor with in vitro and in vivo antitumor activity. Cancer Res 2010 70(1):288-298.

19. Makky K, Tekiela J, Mayer AN: Target of rapamycin (TOR) signaling controls epithelial morphogenesis in the vertebrate intestine. Dev Biol 2007, 303(2):501-513.

20. Akimenko MA, Johnson SL, Westerfield M, Ekker M: Differential induction of four msx homeobox genes during fin development and regeneration in zebrafish. Development 1995, 121(2):347-357.

21. Urasaki A, Morvan G, Kawakami K: Functional dissection of the Tol2 transposable element identified the minimal cis-sequence and a highly repetitive sequence in the subterminal region essential for transposition. Genetics 2006, 174(2):639-649.

22. Thummel R, Burket $C T$, Hyde DR: Two different transgenes to study gene silencing and re-expression during zebrafish caudal fin and retinal regeneration. Sci World J 2006, 6(Suppl 1):65-81.

23. Hoptak-Solga AD, Nielsen S, Jain I, Thummel R, Hyde DR, lovine MK: Connexin43 (GJA1) is required in the population of dividing cells during fin regeneration. Dev Biol 2008, 317(2):541-548.

24. Varga M, Sass M, Papp D, Takács-Vellai K, Kobolak J, Dinnyés A, Klionsky DJ, Vellai T: Autophagy is required for zebrafish caudal fin regeneration. Cell Death Differ 2014, 21(4):547-556.

25. Vlahos CJ, Matter WF, Hui KY, Brown RF: A specific inhibitor of phosphatidylinositol 3-kinase, 2-(4-morpholinyl)-8-phenyl-4H-1-benzopyran-4one (LY294002). J Biol Chem 1994, 269(7):5241-5248.

26. Mitsiades CS, Mitsiades NS, McMullan CJ, Poulaki V, Shringarpure R, Akiyama M, Hideshima T, Chauhan D, Joseph M, Libermann TA, García-Echeverría C, Pearson MA, Hofmann F, Anderson KC, Kung AL: Inhibition of the insulin-like growth factor receptor-1 tyrosine kinase activity as a therapeutic strategy for multiple myeloma, other hematologic malignancies, and solid tumors. Cancer Cell 2004, 5(3):221-230.

27. Chen B, Dodge ME, Tang W, Lu J, Ma Z, Fan CW, Wei S, Hao W, Kilgore J, Williams NS, Roth MG, Amatruda JF, Chen C, Lum L: Small moleculemediated disruption of Wnt-dependent signaling in tissue regeneration and cancer. Nat Chem Biol 2009, 5(2):100-107.

28. Mohammadi M, McMahon G, Sun L, Tang C, Hirth P, Yeh BK, Hubbard SR, Schlessinger J: Structures of the tyrosine kinase domain of fibroblast growth factor receptor in complex with inhibitors. Science 1997 276(5314):955-960.

29. Favata MF, Horiuchi KY, Manos EJ, Daulerio AJ, Stradley DA, Feeser WS, Van Dyk DE, Pitts WJ, Earl RA, Hobbs F, Copeland RA, Magolda RL, Scherle PA Trzaskos JM: Identification of a novel inhibitor of mitogen-activated protein kinase kinase. J Biol Chem 1998, 273(29):18623-18632.

30. ten Freyhaus H, Huntgeburth M, Wingler K, Schnitker J, Bäumer AT, Vantler M, Bekhite MM, Wartenberg M, Sauer H, Rosenkranz S: Novel Nox inhibitor VAS2870 attenuates PDGF-dependent smooth muscle cell chemotaxis, but not proliferation. Cardiovasc Res 2006, 71(2):331-341.

31. Shimobayashi M, Hall MN: Making new contacts: the mTOR network in metabolism and signalling crosstalk. Nat Rev Mol Cell Biol 2014, 15(3):155-162.

32. Kujawski S, Lin W, Kitte F, Börmel M, Fuchs S, Arulmozhivarman G, Vogt S, Theil D, Zhang Y, Antos CL: Calcineurin regulates coordinated outgrowth of zebrafish regenerating fins. Dev Cell 2014, 28(5):573-587.

33. Chablais F, Jazwinska A: IGF signaling between blastema and wound epidermis is required for fin regeneration. Development 2010, 137(6):871-879.

34. Valvezan AJ, Huang J, Lengner CJ, Pack M, Klein PS: Oncogenic mutations in adenomatous polyposis coli (Apc) activate mechanistic target of rapamycin complex 1 (mTORC1) in mice and zebrafish. Dis Model Mech 2014, 7(1):63-71.

35. Inoki K, Ouyang H, Zhu T, Lindvall C, Wang Y, Zhang X, Yang Q, Bennett C, Harada Y, Stankunas K, Wang CY, He X, MacDougald OA, You M, Williams BO, Guan KL: TSC2 integrates Wnt and energy signals via a coordinated phosphorylation by AMPK and GSK3 to regulate cell growth. Cell 2006, 126(5):955-968. 
36. Hirose K, Shimoda N, Kikuchi Y: Transient reduction of 5-methylcytosine and 5-hydroxymethylcytosine is associated with active DNA demethylation during regeneration of zebrafish fin. Epigenetics 2013, 8(9):899-906.

37. Hyde DR, Godwin AR, Thummel R: In vivo electroporation of morpholinos into the regenerating adult zebrafish tail fin. J Vis Exp 2012, Mar 29 (61):e3632.

38. Kim SH, Kowalski ML, Carson RP, Bridges LR, Ess KC: Heterozygous inactivation of tsc2 enhances tumorigenesis in p53 mutant zebrafish. Dis Model Mech 2013, 6(4):925-933.

39. Boglev Y, Badrock AP, Trotter AJ, Du Q, Richardson EJ, Parslow AC, Markmiller SJ, Hall NE, de Jong-Curtain TA, Ng AY, Verkade H, Ober EA, Field HA, Shin D, Shin CH, Hannan KM, Hannan RD, Pearson RB, Kim SH, Ess KC, Lieschke GJ, Stainier DY, Heath JK: Autophagy induction is a Tor- and Tp53-independent cell survival response in a zebrafish model of disrupted ribosome biogenesis. PLoS Genet 2013, 9(2):e1003279.

40. Westerfield M: The zebrafish book. Eugene: University of Oregon Press; 1995.

doi:10.1186/s12861-014-0042-9

Cite this article as: Hirose et al:: Mechanistic target of rapamycin complex 1 signaling regulates cell proliferation, cell survival, and differentiation in regenerating zebrafish fins. BMC Developmental Biology 2014 14:42.

\section{Submit your next manuscript to BioMed Central and take full advantage of:}

- Convenient online submission

- Thorough peer review

- No space constraints or color figure charges

- Immediate publication on acceptance

- Inclusion in PubMed, CAS, Scopus and Google Scholar

- Research which is freely available for redistribution 